\title{
Des shadufs en Alsace
}

Identification de puits à balancier de l'époque romaine

Shadufs in Alsace-Identifying Roman Balance Wells

\section{Olivier Chifflet, Loïc Daverat et Axelle Murer}

\section{(2) OpenEdition}

\section{Journals}

Édition électronique

URL : http://journals.openedition.org/artefact/5973

DOI : $10.4000 /$ artefact. 5973

ISSN : 2606-9245

Éditeur :

Association Artefact. Techniques histoire et sciences humaines, Presses universitaires du Midi

\section{Édition imprimée}

Date de publication : 15 juillet 2020

Pagination : 303-316

ISBN : 978-2-8107-0691-4

ISSN : 2273-0753

\section{Référence électronique}

Olivier Chifflet, Loïc Daverat et Axelle Murer, «Des shadufs en Alsace », Artefact [En ligne], 12 | 2020, mis en ligne le 21 décembre 2020, consulté le 23 décembre 2020. URL : http://

journals.openedition.org/artefact/5973; DOI : https://doi.org/10.4000/artefact.5973

\section{(2) $\Theta \Theta \Theta$}

Artefact, Techniques, histoire et sciences humaines est mise à disposition selon les termes de la Licence Creative Commons Attribution - Pas d'Utilisation Commerciale - Pas de Modification 4.0 International. 


\section{Des shadufs en Alsace}

Identification de puits à balancier de l'époque romaine Olivier Chifflet, Loïc Daverat et Axelle Murer

\section{Résumé}

La fouille du site d'Éguisheim - Lotissement Herrenweg (Haut-Rhin, Alsace) a permis la mise au jour de deux puits gallo-romains. L'identification des fondations de pivots associées a orienté la réflexion sur l'hypothèse de systèmes de puisage à balanciers, technique rarement identifiable. L'utilisation de données statistiques chiffrées a permis à la fois de compléter les données issues du terrain, de confronter la viabilité de notre hypothèse et d'en proposer une restitution à partir des mesures enregistrées lors de la fouille.

\section{Mots-clés}

archéologie, données chiffrées, époque romaine, poids et mesures, statistiques

99 Olivier Chifflet, Loïc Daverat et Axelle Murer, « Des shadufs en Alsace. Identification de puits à balancier de lépoque romaine », Artefact, 12, 2020, p. 303-316. 


\section{Shadufs in Alsace-Identifying Roman Balance Wells}

\section{Abstract}

The archaeological dig of Éguisheim-Lotissement Herrenweg (Haut-Rhin, Alsace) allowed the discovering of two roman wells. The identification of the linked pivots foundations guided our thinking towards the hypothesis of balance drawing systems, hardly identifiable technology. The use of quantified statistical data authorized at once to complete the data of the digging, to confront our hypothesis' viability and to propose a restitution of it, on the basis of the measures recorded during the archaeological dig.

\section{Keywords}

archaeology, quantified data, Roman period, weights and measures, statistics 


\section{Introduction}

Le village d'Éguisheim est situé à $6 \mathrm{~km}$ au sud-ouest de Colmar (Fig. XLVIII, cahier couleur). Il s'appuie sur le versant est du Schlossberg, précédé d'une zone de collines à faible pente dite sous-vosgiennes. Les alentours de la commune sont riches en vestiges archéologiques comme en témoignent les nombreuses découvertes anciennes réalisées à la fin du XIX ${ }^{e}$ et au début du $\mathrm{xx}^{\mathrm{e}}$ siècle, principalement par Karl Gutmann mais également par les exploitants de la glaisière des anciennes tuileries mécaniques situées à quelques dizaines de mètres au nord du terrain investigué en 2015 (Responsable d'opération : Axelle Murer).

Les opérations d'archéologie préventive ayant eu lieu sur le site d'Éguisheim ont été motivées par l'important potentiel archéologique, pour les périodes protohistorique et gallo-romaine, des terrains concernés par la création d'un projet de lotissement (Lotissement Herrenweg). Au total, environ $9220 \mathrm{~m}^{2}$ ont été décapés puis fouillés.

Les vestiges antiques mis au jour correspondent à des installations caractéristiques de fonds de parcelles d'habitat allongées tels que des silos maçonnés, une cave, des bâtiments établis en matériaux légers et des foyers.

Parmi ces vestiges, on distingue plus particulièrement deux puits maçonnés (160 et 355) des II $^{\mathrm{e}}-\mathrm{IV}^{\mathrm{e}}$ siècles de notre ère ${ }^{1}$, qui retiennent notre attention par les aménagements qu'ils présentent. L'un et l'autre présentent des cuvelages maçonnés analogues, et sont associés à de profondes fondations de pivots qui ont pu, dès la phase de terrain, orienter la réflexion vers l'hypothèse de puisages à balanciers, dispositifs encore peu documentés pour la période antique en Gaule - comme en témoignent les plus récents travaux sur le sujet ${ }^{2}$.

La structure 355 a fait l'objet d'une reconstitution, puisque mieux préservée que le puits 160 ; elle a en effet livré un niveau de comblement inférieur particulièrement humide, probable indicateur de la proximité des niveaux phréatiques, ainsi que la partie sommitale du cuvelage comprenant de larges dalles appartenant à la margelle (Fig. XLIX-a, cahier couleur).

1. Datations obtenues par l'étude du mobilier céramique mis au jour dans les comblements de ces structures.

2. Voir notamment Coadic, 2009. 
Précisons que le cuvelage de ce puits est constitué de blocs grossièrement équarris et de dalles brutes, décimétriques, en opus incertum. Dans le second palier de fouille du cuvelage, nous avons pu observer de l'argile fine en plaquettes entre les blocs. En outre, le comblement du cuvelage est à ce niveau particulièrement glaiseux. Il est probable que les blocs du cuvelage aient été jointés avec de l'argile glaiseuse, dont les plaquettes observées constituent les vestiges. Ce puits a fonctionné avec la fosse de fondation 365 destinée à caler le madrier du balancier (Fig. L, cahier couleur, Fig. 1)

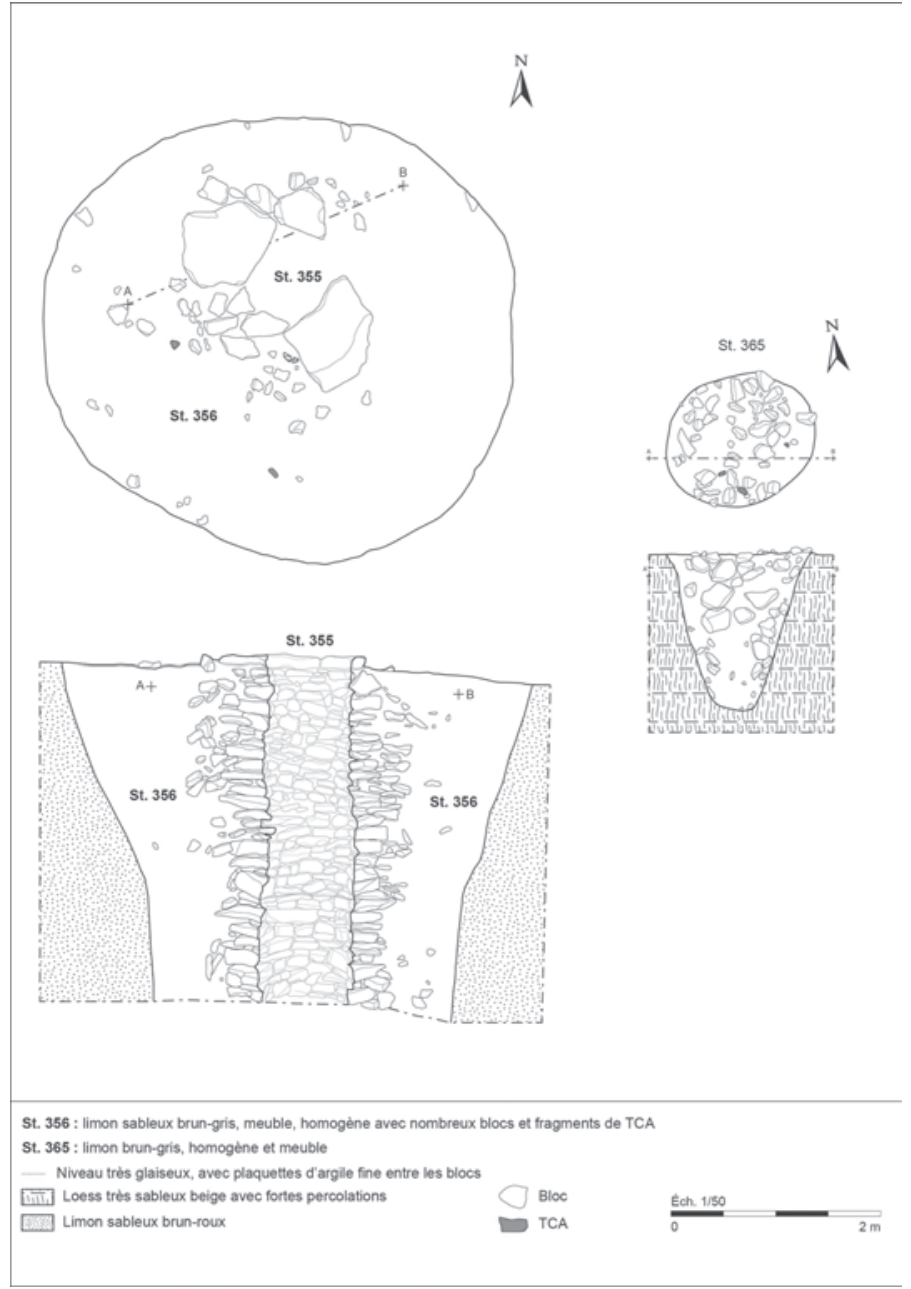

Fig. 1. - Relevés d'architecture en plan et en coupe du puits 355 et de la fondation de pivot 365

Dessins Fr. Bachellerie, L. Daverat ; DAO L. Daverat, C. Ecarot. 


\section{Démarche et contraintes}

Toutes périodes chrono-culturelles confondues, les puits ne constituent pas, en eux-mêmes, des découvertes rares en archéologie, et ce quel qu'en soit le contexte. À l'époque gallo-romaine, ils sont souvent maçonnés, cet appareillage pouvant largement être étudié, ainsi que la profondeur de leur creusement, dans la limite des contraintes de prescription.

Cependant, leur état de conservation ne concerne le plus souvent que la substruction proprement dite, la structure hors sol (et donc le système de puisage) demeurant inconnue. De rares mises au jour permettent parfois d'identifier ce système de puisage lorsque des éléments sont retrouvés préservés en contexte anaérobique dans le comblement du puits. Cela n'est toutefois pas le cas du puits 355 du Lotissement Herrenweg. Seule l'identification, dans l'environnement immédiat du puits proprement dit, d'une fondation ayant pu permettre l'installation d'un pivot de système de balancier nous permettait d'envisager une restitution de ce dernier.

De fait, l'approche strictement archéologique nécessitait d'être complétée par une démarche plus technique.

La plupart du temps, si la qualité scientifique exige une réflexion poussée sur le site autant qu'une remise en contexte au moins local, les données techniques du domaine hydraulique sont malheureusement délaissées de l'archéologie. Cette documentation existe pourtant, même si elle n'a que très faiblement pénétré le champ de l'archéologie, à l'occasion - rare de problématiques particulières. À plus forte raison, la bibliographie qui accompagne le présent article met l'accent sur la carence d'études de synthèse sur ce point en Alsace.

Toutefois, nous remarquons l'existence de plusieurs travaux de recherche hors du champ de l'archéologie et qui peuvent s'avérer précieux pour l'appréhension des modalités techniques de puisage en vue d'éventuelles reconstitutions. Ces études appartiennent généralement au domaine de la préservation du patrimoine régional récent et concernent particulièrement les systèmes de puisage à balancier, parfois encore en activité jusque dans les années $1970^{3}$.

3. Nous ferons notamment appel, ici, à Lassure, 1986. 
Si le système de balancier du puits 355 est archéologiquement attesté, on en trouve notamment une représentation de l'époque romaine parmi les mosaïques du site tunisien d'Oudna ${ }^{4}$, les données nous manquent pour en estimer précisément les dimensions et les proportions. En effet, celles-ci sont soumises non seulement à des rapports fonctionnels particuliers mais encore à des variabilités parfois importantes lors de leur mise en place (liées à la spécialisation ou non de l'artisan, aux matériaux mis en œuvre, etc. $)^{5}$. Parmi les données importantes qui nous manquent en ce qui concerne le puits 355, citons en particulier la hauteur hors sol du pivot. Nous ne pouvons fonder notre raisonnement que sur deux données objectives livrées par la fouille : le diamètre du cuvelage du puits $(0,80 \mathrm{~m})$ et la distance théorique du centre de la fondation du pivot ${ }^{6}$ et du centre intérieur du puits (environ 5,44 m). À partir de cela, plusieurs études de puits à balanciers nous permettent tout de même d'en proposer une restitution, circonscrite dans des fourchettes de dimensions et de proportions à la fois fonctionnelles et plausibles eu égard aux vestiges mis au jour.

En effet, le puits à balancier est un système de puisage en lui-même bien connu et encore largement employé dans plusieurs pays d'Afrique de l'Est et des Proche et Moyen-Orient, où il porte communément le nom de shaduf. Charles Hiégel explique cette popularité et cette pérennité par la simplicité du mécanisme en question :

Le fonctionnement du puits à balancier était d'une extrême simplicité. Une grosse poutre en bois de chêne, comportant à son sommet une entaille qui permettait le passage d'une longue perche de bois, était enfoncée verticalement dans le sol à quelques mètres du puits. La perche, dont la section allait en s’amenuisant du côté où l'on puisait l'eau, avait deux bras inégaux. Le bras le plus court était muni d'un contrepoids, pierre ou morceau de bois, tandis qu'au bras le plus long était fixée une longue tige de bois à laquelle on accrochait un seau au moyen d'une chaîne. Par un mouvement

\footnotetext{
4. Mosaïque de l'atrium de la villa des Laberii, conservée au Musée National du Bardo. Il n'est pas inintéressant de noter que, dans cette représentation des activités agricoles, le puits occupe une place centrale dans la composition.

5. Lassure, 1986.

6. En justification de ce choix centré, rappelons que le probable négatif du pivot proprement dit est lisible dans le comblement de sa fondation, en position effectivement centrale (Fig. L, cahier couleur).
} 
de la main on abaissait la tige dans le puits ; celle-ci par l'effet du contrepoids se relevait ensuite sans aucune difficulté jusqu’à la margelle du puits avec le seau rempli d'eau' .

\section{Proposition de restitution du puits 355 La margelle}

La margelle porte des traces d'usure très marquées (Fig. XLIX-a, cahier couleur). La question se pose de savoir s'il y a eu ou non une margelle en élévation sur ces dalles, au moins en matériaux périssables. Rien ne le prouve (aucune trace d'usure significative). On peut tout aussi bien imaginer une margelle surélevée en bois qu'une ouverture de puits à l'identique de celle mise au jour, sans autre aménagement. De plus, le système de puisage à balancier pourrait plaider autant pour cette hypothèse (réduire la hauteur nécessaire de levage du récipient répond à une considération pratique) que pour une margelle en élévation (la hauteur du pivot pourrait être suffisante, et les aspects de sécurité ne sont pas absents des aménagements antiques).

\section{Niveau de l'eau}

La présence de puits à balancier sur le site dément la tradition locale qui voudrait que la nappe phréatique se trouve, à ce niveau du piémont vosgien d'Éguisheim, au minimum à $8 \mathrm{~m}$ de profondeur ${ }^{8}$. La réalité pédologique n'est certainement pas aussi homogène. En effet, le choix stratégique qui consiste à mettre en œuvre un système de puisage particulier n'est pas innocent, et ainsi les puits à balancier sont conçus pour des nappes phréatiques peu éloignées du niveau du sol :

Ce sont les systèmes de puisage qui différencient les puits ; mais ils sont eux-mêmes fonction du contexte pédologique. Ainsi en va-t-il des puits à balancier : de la longueur de leur flèche, et de leur

\footnotetext{
7. Hiégel, 1987 , p. 244-245. L'auteur précise en outre que « le puits à balancier peut prendre dans les textes les noms latins de furca (fourche, potence) en Lorraine ou pertica (perche), plus rare en Lorraine mais commun en Franche-Comté (et que l'on y trouve également francisé) ».

8. Témoignages concordants des différents exploitants voisins rencontrés lors de l'intervention sur le terrain.
} 
hauteur au-dessus de la margelle, dépend la profondeur atteinte par le seau. Pour des raisons de contraintes physiques du matériau, ces puits ne peuvent guère dépasser les six mètres de haut. Plus grands, ils sont trop lourds à manœuvrer. Ils conviennent donc aux nappes phréatiques proches du niveau du sol. Son adaptation au contexte hydrographique explique qu'il s'en trouve partout où la nappe est proche du sol. Il ne faut voir dans ce phénomène nul effet d'une influence esthétique ou artistique, nul topos - mais l'exact reflet d'une réalité encore observable dans l'ethnographie aujourd'hui'.

$\mathrm{Si}$, comme nous l'avons dit, les puits à balancier sont assez difficilement identifiés en archéologie, l'étude des différents exemples encore présents dans le paysage contemporain corrobore largement cette assertion. Ainsi, l'étude de deux puits à balancier de la commune de Sorges (Dordogne) par le Conseil d'Architecture, d'Urbanisme et d'Environnement ${ }^{10}$ recense des niveaux d'eau à $2,50 \mathrm{~m}$ (lieu-dit Fontnovias) et 2,30 m (lieu-dit Pouzalimont) du bord de chacun des puits. De la même manière, une étude technique et statistique très détaillée de 29 shadufs encore en activité dans le Kanem (Chad) conclut à une moyenne du niveau d'eau à 3,84 $\mathrm{m}$ du bord des puits, avec une déviation standard de $1,50 \mathrm{~m}^{11}$. Si cette déviation peut indiquer des niveaux d'eau jusqu'à $5,34 \mathrm{~m}$, précisons que de telles mesures ne concernent que des puits à balancier dont le fonctionnement est assisté par motorisation ; ceux encore actionnés manuellement ont les rapports niveau d'eau/margelle les plus modestes ${ }^{12}$.

Ainsi, le comblement du puits 355 devient particulièrement glaiseux à partir d'environ 2,50 $\mathrm{m}$ de profondeur (Fig. L, cahier couleur), et ce de manière très marquée. Si cela n'indique probablement pas le niveau antique de l'eau, nous pouvons en déduire une certaine proximité de la nappe phréatique et un rapport niveau d'eau/margelle qui s'inscrit bien dans les dimensions modestes associées aux puits à balancier. Nous admettrons par conséquent une distance niveau d'eau/margelle située entre 2,50 et $4 \mathrm{~m}$, en appliquant précisément la déviation standard de 1,50 m observée sur les

9. Alexandre-Bidon, 1992, p. 528.

10. Eu égard à la fois à l'aspect purement technique de ce développement et à la carence dans les études de puits à balancier strictement alsaciens, il apparaît pertinent de faire appel à des points de comparaison hors région. Robert, Watteau, 2013.

11. Mirti et al., 1999, p. 228.

12. Mirti et al., 1999, p. 228-229. 
shadufs aux observations effectuées sur le comblement du puits 355. Cette estimation est concordante avec le sondage géotechnique RG2, publié dans le rapport de diagnostic de l'Inrap ${ }^{13}$, qui a permis d'identifier le niveau de la nappe phréatique à $4 \mathrm{~m}$ environ.

\section{Dimensions des éléments verticaux}

Le tableau ci-dessous (Fig. 2) se fonde principalement sur la publication de Mirti et al. ${ }^{14}$, qui est à la fois la plus détaillée sur le plan technique (il s’agit de travaux d'ingénierie hydraulique), la plus représentative sur le plan statistique (étude exhaustive de 29 shadufs) et la plus prudente quant aux résultats présentés (établissement de mesures moyennes avec précision systématique de la mesure de déviation standard correspondante). Par souci d'exhaustivité, nous avons en outre voulu intégrer à ce tableau les observations des dossiers d'inventaire architectural de deux puits à balanciers français ${ }^{15}$.

Ce tableau distingue deux parties : les données utiles des études statistiques, hydrauliques et architecturales d'une part (à gauche, fond blanc) et l'application de ces rapports statistiques au puits 355 d'autre part (à droite, fond coloré).

13. Griselin et al., 2014, p. 39, fig. 12.

14. Mirti et al., 1999.

15. Les deux exemples en question sont périgourdins - il n'existe malheureusement aucun dossier d'inventaire architectural régionalement plus pertinent. Robert, Watteau, 2013. 
Fig. 2. - Tableau des calculs de restitution des dimensions du mécanisme de balancier du puits 355

\begin{tabular}{|c|c|c|c|c|c|c|}
\hline \multirow{3}{*}{ Référence } & \multicolumn{3}{|c|}{$\begin{array}{l}\text { Données des études statistiques, } \\
\text { hydrauliques et architecturales }\end{array}$} & \multicolumn{3}{|c|}{$\begin{array}{l}\text { Application des rapports statistiques } \\
\text { au puits } 355 \text { d'Eguisheim Lotissement } \\
\text { Herrenweg }\end{array}$} \\
\hline & \multicolumn{2}{|l|}{ Mirti et al. } & \multirow{2}{*}{$\begin{array}{l}\text { Robert, } \\
\text { Watteau } \\
(\text { CAUE) }\end{array}$} & \multirow{2}{*}{$\begin{array}{l}\text { Calcul } \\
\text { distance } \\
\text { pivot-puits/ } \\
\text { Mirti et al. }\end{array}$} & \multirow{2}{*}{$\begin{array}{l}\text { Fourchette } \\
\text { avec } \\
\text { déviation } \\
\text { standard } \\
\text { Mirti } \text { et al. }\end{array}$} & \multirow{2}{*}{$\begin{array}{l}\text { Calcul } \\
\text { distance } \\
\text { pivot-puits } \\
\text { CAUE }\end{array}$} \\
\hline & $\begin{array}{l}\text { Mesure } \\
\text { moyenne }\end{array}$ & $\begin{array}{l}\text { Déviation } \\
\text { standard }\end{array}$ & & & & \\
\hline $\begin{array}{l}\text { Niveau } \\
\text { statique } \\
\text { de leau }\end{array}$ & 3,84 & 1,50 & $2,30-2,50$ & $\begin{array}{l}2,50-4^{\mathrm{b}} \\
(\text { calcul } \\
6,3462)\end{array}$ & $\begin{array}{l}2,50-4 \\
\text { (calcul } \\
4,8462- \\
7,8462)\end{array}$ & $\begin{array}{l}3,7867- \\
4,1160 \\
(\max \\
3,7892)\end{array}$ \\
\hline $\begin{array}{l}\text { Longueur } \\
\text { de corde }\end{array}$ & 5,22 & 1,42 & & 8,6269 & $\begin{array}{l}7,2069- \\
10,0469 \\
\end{array}$ & \\
\hline $\begin{array}{l}\text { Distance } \\
\text { pivot/puits } \\
\text { (de centre } \\
\text { à centre) }\end{array}$ & $3,2925^{c}$ & & $\begin{array}{l}\text { 3,02-3,59 } \\
\text { (moyenne } \\
3,305)\end{array}$ & 5,4414 & & \\
\hline $\begin{array}{l}\text { Diamètre } \\
\text { cuvelage }\end{array}$ & 1,025 & 0,19 & $0,90-0,95$ & 0,80 & & \\
\hline Hauteur pivot & 3,11 & 0,74 & $4,5-5$ & 5,1397 & $\begin{array}{l}4,3997- \\
5,8797\end{array}$ & $\begin{array}{l}7,4088- \\
8,2320 \\
(\max . \\
7,5785) \\
\end{array}$ \\
\hline $\begin{array}{l}\text { Longueur } \\
\text { du fléau }\end{array}$ & $4,7^{\mathrm{d}}$ & 1,1 & 10 & 7,7675 & $\begin{array}{l}6,6675- \\
8,8675\end{array}$ & $\begin{array}{l}16,4641 \\
(\max . \\
15,1571)\end{array}$ \\
\hline $\begin{array}{l}\text { Longueur } \\
\text { avant du fléau }\end{array}$ & 2,78 & 0,88 & & 4,5944 & $\begin{array}{l}3,7144- \\
5,4744\end{array}$ & \\
\hline $\begin{array}{l}\text { Longueur ar- } \\
\text { rière du fléau }\end{array}$ & 1,92 & 0,22 & & 3,1731 & $\begin{array}{l}2,9531- \\
3,3931\end{array}$ & \\
\hline
\end{tabular}

a CAUE : Conseil d'architecture, d'urbanisme et d'environnement de la Dordogne - organisme de publication des deux dossiers d'inventaire architectural, Robert, Watteau, 2013.

${ }^{b}$ Les mesures présentées sur fond plus foncé sont issues des observations faites sur le terrain et qui servent par conséquent de bases tangibles à notre proposition de restitution.

'Cette mesure n'est pas fournie telle quelle par l'étude de Mirti et al., nous l'avons calculée de la manière suivante d'après leurs données : Front lever length + Extension length + (Welldiameter/2). Mirti et al., 1999, p. 228. Nous nous sommes toutefois abstenus, par précaution, de calculer la déviation standard correspondante.

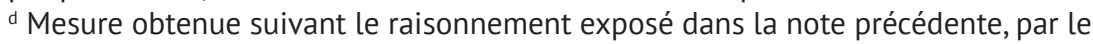
calcul : Rear lever length + Front lever length + Extension length. Cette mesure permet la comparaison avec les observations de Robert, Watteau, 2013. 
Les observations réalisées sur le terrain (estimation du niveau statique de l'eau, distance pivot/puits, diamètre du cuvelage) aboutissent, d'après les données de Mirti et al., à des résultats qui appellent quelques remarques :

- plusieurs éléments correspondent bien aux fourchettes des travaux de Mirti et al. lorsque l'on prend en compte les mesures de déviation standard qui s'y rapportent : niveau statique de l'eau $(2,50 / 4 \mathrm{~m}$ pour le puits 355 contre 1,34/5,34 $\mathrm{m}$ chez Mirti et al.), diamètre du cuvelage ( $0,80 \mathrm{~m}$ contre $0,81 / 1,20 \mathrm{~m})$;

- sous forme de moyennes, les résultats obtenus demeurent cohérents : la hauteur du pivot $(5,13 \mathrm{~m})$ est comparable à celles observées par Nicole Robert et Bernard Watteau (4,5/5 m) ; la longueur totale du fléau $(7,76 \mathrm{~m})$ entre dans une fourchette qui comprend les données de Mirti et al. (3,60 m) et celles de Robert et Watteau $(10 \mathrm{~m})$;

- les autres résultats obtenus demeurent cohérents en tant qu'extrêmes inférieurs avec application de la déviation standard en positif : longueur de corde de 7,20 m contre 6,64 m chez Mirti et al.; longueur avant du fléau de 3,71 m contre 3,66 m chez Mirti et al.

Les calculs effectués à partir des travaux de Mirti et al. permettent de restituer un puits à balancier comparable aux plus imposants de leur référentiel (Fig. 3). Nous notons en revanche que les mêmes calculs, effectués d'après les données de Robert et Watteau aboutissent à des résultats démesurés (fléau de plus de $16 \mathrm{~m}$, hauteur de pivot de plus de $7 \mathrm{~m}$ ), qui n'entrent pas même dans les ordres de mesure de leurs observations. Paradoxalement, ces divergences de résultats constituent un argument, encore, en faveur de notre proposition de restitution : non seulement cette dernière se situe dans les ordres de grandeur des rapports dynamiques fonctionnels observés par Mirti et al., mais encore les variations considérables de ces mêmes rapports dynamiques entre la base de données de Mirti et al. et les observations de Robert et Watteau (d'où les grandes différences de résultats) démontrent l'importante variabilité de ces mêmes rapports dynamiques pour des puits à balancier pourtant bien tous fonctionnels. 


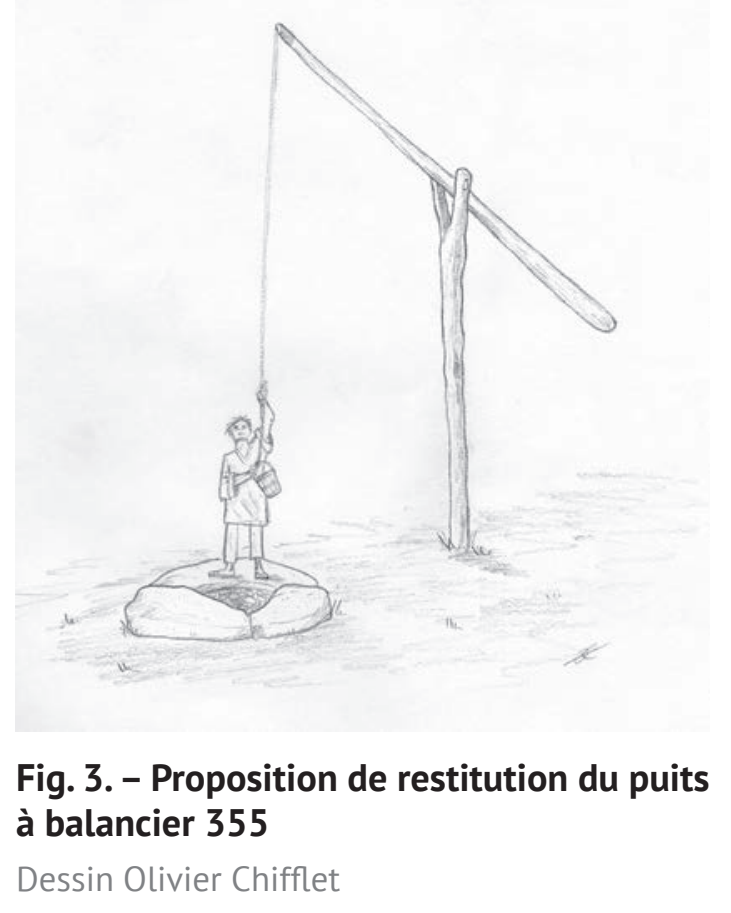

\section{Conclusion}

L'identification de puits à balanciers sur le site d'Éguisheim - Lotissement Herrenweg représente en soi un apport certain, du fait de la difficulté à en collecter les arguments sur le terrain. De fait, et comme nous l'avons dit, aucun élément de puisage n'a été mis au jour lors de la fouille des deux puits concernés. Toutefois, la lisibilité de la corrélation entre le puits 355 et la fondation du pivot associé nous a bel et bien permis de reconnaître un système de puisage à balancier.

L'utilisation de données statistiques chiffrées nous autorise aujourd'hui à en proposer une restitution, autant qu'à vérifier comparativement la validité de notre hypothèse.

Cette approche mérite d'être reconduite lorsque les données mises au jour sur le terrain le permettent. Ainsi, les données techniques de chaque structure ou fait considérés (puits proprement dit, niveau phréatique, aménagements associés...) doivent être confrontées à des données statistiques chiffrées afin de vérifier la viabilité de toute hypothèse envisagée. 


\section{Bibliographie}

Alexandre-Bidon Danièle, "Archéo-iconographie du puits au Moyen Âge (XII ${ }^{\mathrm{e}}$ $\mathrm{xvI}^{\mathrm{e}}$ siècles) ", Mélanges de l'École française de Rome. Moyen Âge. Temps modernes, t. $104, n^{\circ} 2,1992$, p. 519-543.

CoAdic Sophie, Les machines d'élévation dans le monde romain du II siècle av. J.-C. au VIe siècle apr. J.-C., thèse de doctorat, Bordeaux, 2009.

Griselin Sylvain (dir.) et al., Éguisheim (Haut-Rhin), Unterer Teil vomHerrenweg - lotissement Herrenweg : des occupations gallo-romaines et protohistoriques, rapport de diagnostic archéologique. Strasbourg, Inrap Grand Est sud, 2014.

Gutmann Karl, "Fundberichte und kleinereNotizen. Die archäologischen Funde von Éguisheim (1888-1898) », Bulletin de la Société pour la Conservation des Monuments Historiques d'Alsace, vol. II, 20, 1902, p. 1-87.

Hiégel Charles, "Du puits à balancier aux pompes. L'élévation de l'eau salée dans les salines Lorraines du Moyen Âge au XviII e siècle ", Les Cahiers Lorrains, n 3 1987, p. 243-285.

LASSURE Christian, Une vieille technique de puisage en perdition : le balancier à tirer l'eau. CERAV, Études et recherches d'architecture vernaculaire, Paris, ${ }^{\circ} 6$, 1986.

Mirti T.H., Wallender Wesley W., Chancellor W.J., Grismer Mark E., "Permormance characteristics of the shaduf: A manual water-lifting device", Applied Engineering in Agriculture, 15 (3), 1999, p. 225-231.

Murer Axelle et al. (dir.), Éguisheim - Lotissement Herrenweg (Alsace, Haut-Rhin, 68), rapport final d'opération d'archéologie preventive, Habsheim : Antea Archéologie, 2016, 2 volumes.

Robert Nicole, Watteau Bernard, Dossier d'inventaire petit patrimoine rural bâti du Périgord. Sorges, lieu-dit Fontnovias, dossier d'inventaire architectural. Périgueux : Conseil d'architecture, d'urbanisme et d'environnement de la Dordogne (CAUE 24), 2013.

Robert Nicole, Watteau Bernard, Dossier dinventaire petit patrimoine rural bâti du Périgord. Sorges, lieu-dit Pouzalimont, dossier d'inventaire architectural. Périgueux : Conseil d'architecture, d'urbanisme et d'environnement de la Dordogne (CAUE 24), 2013. 
Olivier Chifflet, Loïc Daverat et Axelle Murer

\section{Les auteurs}

Olivier Chifflet est archéologue, Dessin de mobilier et de restitution, Antea Archéologie.Contact : olivier.chifflet@antea-archeologie.com

Loïc Daverat est responsable d’opération (Antiquité), Archéogéographie, Antea Archéologie.Contact : loic.daverat@antea-archeologie.com

Axelle Murer est responsable d'opération (Antiquité), Céramologie, Antea Archéologie.Contact : axelle.murer@antea-archeologie.com 